\title{
Malignant Granulosa Cell Tumor
}

National Cancer Institute

\section{Source}

National Cancer Institute. Malignant Granulosa Cell Tumor. NCI Thesaurus. Code C4205.

A granulosa cell tumor which has an aggressive clinical course and metastasizes to other anatomic sites. 